\section{Low-Temperature AFM Reveals Hidden Atom in Graphite Surface}

The existence of atoms was hotly debated by leading scientists only 100 years ago. While field ion microscopy was the first technique to image individual atoms, scanning tunneling electron microscopy (STM) is now routinely used to observe single atoms in real space on flat surfaces. However, a classic problem is the "missing" atoms in the surface of graphite; that is, the three atoms $(\alpha)$ in the hexagonal lattice bonded to subsurface atoms have less electron density at the Fermi level than the three atoms $(\beta)$ lying above holes. Because STM only probes the mobile electrons that cause the electric conductivity - that is, the electrons with an energy close to the Fermi level-only $\beta$ atoms appear in STM images. Although atomic force microscopy (AFM) should in principle be able to image all surface atoms, previous AFM images showed only a single protrusion per unit cell. Recently, however, S. Hembacher, F.J. Giessibl, and J. Mannhart of Universität Augsburg, Germany, and C.F. Quate of Stanford University have shown that the missing surface atoms in graphite are observable with an AFM capable of detecting very small repulsive forces between single atoms.

As reported in the October 28, 2003 issue of the Proceedings of the National Academy of Sciences, the researchers combined STM and AFM at low temperature to simultaneously record the tunneling current and short-range forces to probe the charge density at the Fermi level and the total charge density, respectively, of graphite. Built on a 30-ton foundation to damp external vibrations and immersed in a liquid He bath cryostat, the combined STM-AFM operates in an ultrahigh vacuum with a sample temperature of $4.89 \mathrm{~K}$. The frequency modulation force microscopy method makes possible combined STM-AFM experiments. The simultaneous measurement of current and force allows the research team to rule out double tip effects that probably have caused the sporadic previous observations of the full hexagon in STM or AFM experiments. The researchers had previously achieved subatomic resolution with this technique and imaged structures related to atomic orbitals.

The researchers said that because the layers in graphite are only weakly coupled, the normal forces between the AFM tip and the sample must be kept extremely small in order to avoid distorting the graphite lattice. The tungsten tip was shaped by field emission and controlled collisions until topographic images with corrugation of only $20 \mathrm{pm}$ were obtained.
Whereas an STM image recorded in constant-height mode shows only the $\beta$ atoms, both types of atoms are imaged when the frequency-shift data are recorded simultaneously, that is, with the STMAFM. The researchers attribute the shortrange forces acting between the tip and the sample, indicated by the positive frequency shifts, to overlap of the $5 s-, 5 p-$, and $6 s$-like orbitals of the tungsten tip with the sample orbitals.

Giessibl and co-workers have demonstrated an additional capability of AFM to gather information on surfaces that are only partially accessible by STM. They believe that "the successful imaging of the complete surface lattice of graphite is an important milestone for the imaging of other soft materials, [including] insulating organic molecules, with atomic resolution."

SteVEn TrohalaKI

\section{Pyroelectric Effects Observed in Amorphous $\mathrm{BaTiO}_{3}$}

The pyroelectric effect, in which a temperature change is accompanied by a change in the polarization state of a material, is usually associated with polar crystalline solids. Recent research has focused on size effects, or the reduction of material response at nanometer-size length scales, that may limit device applications. In studying the size limits of crystallites yielding the desired material response, it is interesting to consider whether or not pyroelectric behavior can be observed in noncrystalline materials. In fact, all that is necessary for the manifestation of a pyroelectric response is directionally ordered polar molecules. This can in principle be achieved in a liquid, as evidenced in nematic liquid crystals, as well as in ionic solids. What is needed is a driving force for forming, and then ordering, the polar regions. V. Lyahovitskaya of the Department of Materials and Interfaces at the Weizmann Institute of Science in Israel and co-workers at the Weizmann Institute and the Swiss Federal Institute of Technology have recently produced quasi-amorphous $(<0.3 \%$ crystallinity) solid $\mathrm{BaTiO}_{3}$ exhibiting pyroelectric coefficients of $10-30 \mu \mathrm{C} / \mathrm{m}^{2} \mathrm{~V}$ (5-15\% of singlecrystal values). This work demonstrates the feasibility of producing materials that are pyroelectric while retaining their amorphous character, as evidenced by $\mathrm{x}$-ray diffraction (XRD) and transmission electron microscopy (TEM).

As reported in the November 2003 issue of Advanced Materials, pyroelectrically active $\mathrm{BaTiO}_{3}$ films were obtained by passing as-deposited (rf-sputtered) $\mathrm{BaTiO}_{3}$ films on doped $\mathrm{Si}(100)$ substrates through a steep temperature gradient.
The as-deposited films gave no pyroelectric signal and possessed a temperatureindependent low dielectric constant of $\sim 9$, while the films that passed through the temperature gradient displayed pyroelectric coefficients of $10-30 \mu \mathrm{C} / \mathrm{m}^{2} \mathrm{~K}$, and an increased but essentially temperatureand frequency-independent dielectric constant of 33 with low loss levels of 0.02 . The increase in dielectric constant is consistent with the ordering of crystal motifs. The sign or magnitude of the pyroelectric response did not change after application of a poling field of $200 \mathrm{kV} / \mathrm{cm}$, which indicates a fixed direction of polarization. The researchers reported that additional heat treatments through a temperature gradient, as well as isothermal heating, also did not affect the pyroelectric properties. In addition, films that were passed through the steep temperature gradient displayed piezoelectric activity with $d_{33}$ values of $10 \mathrm{pm} / \mathrm{V}$ (5-10\% of single crystal values). Crystallites were rarely detected by TEM investigations and absent from the XRD spectra, and the concentrations were estimated to be well below $0.3 \%$. Therefore, the observed pyroelectric and piezoelectric activities of up to $15 \%$ of single-crystal values were attributed to the gradient of the strain-induced directional polar state.

KYLE BRINKMAN

\section{Daylight Photocatalysis Achieved on Carbon-Doped $\mathrm{TiO}_{2}$}

The evidence of photoelectrochemical splitting of $\mathrm{H}_{2} \mathrm{O}$ over $\mathrm{TiO}_{2}$ to form $\mathrm{OH}$ radicals by Fujishima and Honda in 1972 initiated a great deal of research activity continuing to the present time. However, the industrial application of titanium dioxide in photocatalysis is still limited because of the large energy bandgap of $\mathrm{TiO}_{2}(3.20 \mathrm{eV})$ that results in absorbance of only a small UV fraction of solar light (about 2-3\%). Nonetheless, S. Sakthivel and $\mathrm{H}$. Kisch from the Institute of Inorganic Chemistry of Erlangen-Nürnberg University, Germany, have recently achieved a significant photodegradation of water pollutants by daylight excitation of carbon-modified titanium dioxide.

As reported in the October 20, 2003 issue of Angewandte Chemie, the researchers synthesized carbon-doped $\mathrm{TiO}_{2}$ by hydrolysis of titanium tetrachloride with tetrabutylammonium hydroxide followed by calcinations in the air. This method leads to the formation of anatasebased compounds that contain residual carbon instead of nitrogen. The researchers determined the band structure of the materials and found the presence of surface states close to the valence-band 